\title{
Redo cardiac surgery in adults
}

\author{
VR Machiraju • Hartzell V Schaff • Lars G Svensson
}

Published online: 2 October 2010

(C) Indian Association of Cardiovascular-Thoracic Surgeons 2010

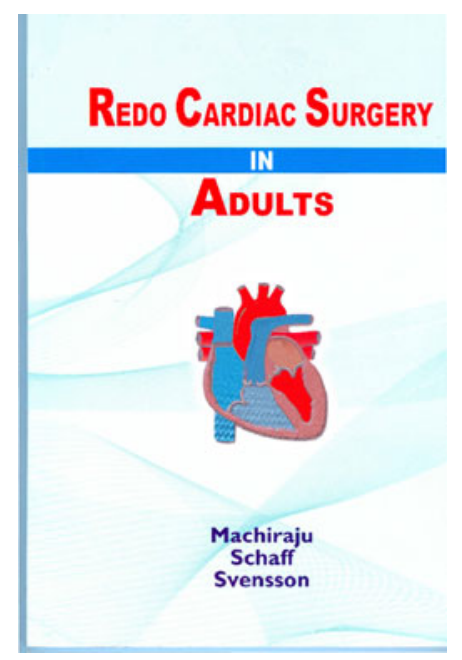

Machiraju, Schaff and Svensson have put together the third edition of this monograph containing several presentations on Redo cardiac surgery in adults. All three editors are highly experienced and carry with them enormous technical ability. Several others have also contributed to this book. It is important to note that these experiences mainly come from The Mayo Clinic, The Cleveland Clinic and The University of Pittsburgh, all of whom perform large number of surgeries including Redo surgeries.

The book has 19 chapters on various aspects of Redo cardiac surgery in adults. They describe the technique and results in Redo coronary surgery, Redo valve surgery, Redo congenital surgery and Redo aortic surgery. The technical

details are clearly described and the reader will find several maneuvers for safe Redo surgery.

The technique of Redo sternotomy has been very well described. The use of an oscillating saw and technical maneuvers to avoid serious hemorrhage during sternal reentry provide the reader with appropriate instructions. The chapter on transfusion and bleeding management as well as anesthesia emphasise team work and indicate the need for additional preparation for redo surgery. The chapters on Redo surgery following previous valve procedure is especially illustrative of safe and effective techniques for reducing both mortality and complications. The chapter on Aortic surgery especially on endovascular treatment clearly demonstrates modern techniques in the management of patients with Aortic disease.

Overall this book would certainly benefit young surgeons who will inevitably perform many of these procedures in their lifetime. The individual case notes and reports are especially helpful. There is not enough emphasis on the technical aspects of primary surgery that will make Redo surgery a lot easier and safe. The avoidance of pledgeted sutures for the ascending Aorta and closure of pericardium to cover the heart and the grafts has not been effectively stressed. The authors may provide indications and techniques to establish femorofemoral bypass, and its advantages in difficult or emergent situations. The surgical photographs need to be improved in quality.

The book will serve the important purpose of educating students of cardiovascular surgery and the young and busy cardiac surgeons to overcome the difficulty and consequences of high mortality and morbidity in Redo cardiac surgery.

A. Sampath Kumar 